\title{
Exponential stability of fractional stochastic differential equations with distributed delay
}

\section{Li Tan}

"Correspondence: tltanli@126.com School of Statistics, Jiangxi University of Finance and Economics, Nanchang, Jiangxi 330013, China

\section{Springer}

\begin{abstract}
Equations driven by fractional Brownian motion are attracting more and more attention. This paper considers fractional stochastic differential equations with distributed delay. With the variation-of-constants formula, an explicit expression and asymptotic behavior of the solution are provided, sufficient conditions are derived to guarantee the $p$ th moment exponential stability and almost surely exponential stability.
\end{abstract}

MSC: 39B82; 60H05

Keywords: fractional Brownian motion; variation-of-constants formula; exponential stability; distributed delay

\section{Introduction}

Fractional Brownian motion (fBm) was first studied in 1940 by Kolmogorov [1], who defined it in a Hilbert space and named it a Wiener helix. Until 1968, Mandelbrot and Van Ness [2] provided the stochastic integral representation of $\mathrm{fBm}$ in terms of a standard Brownian motion, the name $\mathrm{fBm}$ was then introduced. As an extension of classic Brownian motion, $\mathrm{fBm}$ can give a better description to model natural situations like the temperature at a specific place as a function of time and so on [3]. Because of their extensive applications in finance, economics, biology, etc., $\mathrm{fBm}$ is attracting more and more attention.

An $\mathrm{fBm}$ with Hurst index $H \in(0,1)$ is a continuous and centered Gaussian process $\left\{B^{H}(t), t \geq 0\right\}$ with covariance function

$$
E\left[B^{H}(t) B^{H}(s)\right]=\frac{1}{2}\left(t^{2 H}+s^{2 H}-|t-s|^{2 H}\right) .
$$

For $H=\frac{1}{2}$, the $\mathrm{fBm}$ is exactly the standard Brownian motion without memory. For $H \neq \frac{1}{2}$, it is usually divided into two cases, $0<H<\frac{1}{2}$ and $\frac{1}{2}<H<1$. The case for $H>\frac{1}{2}$ is called a long memory process, while $H<\frac{1}{2}$ is called a short memory process.

It is known that $\mathrm{fBm}$ shares something with Brownian motion, but the critical difference is that the increments of $\mathrm{ABm}$ are dependent while that of Brownian motion are independent. Therefore, $\mathrm{fBm}$ is neither a Markov process, nor a semimartingale, and the theories of stochastic differential equations (SDEs) driven by standard Brownian motion are invalid in the case of $\mathrm{fBm}$. Recently, there are some studies on existence, uniqueness, and stability of the solutions for fractional SDEs. Ferrante and Rovira [4] proved the existence

○2014 Tan; licensee Springer. This is an Open Access article distributed under the terms of the Creative Commons Attribution License (http://creativecommons.org/licenses/by/2.0), which permits unrestricted use, distribution, and reproduction in any medium, provided the original work is properly cited. 
and uniqueness for a stochastic delay differential equation driven by a fractional Brownian motion with Hurst parameter $H>1 / 2$. Ferrante and Rovira [5] gave a proof of the existence and uniqueness for stochastic delay differential equations with hereditary drift driven by a fractional Brownian motion with Hurst parameter $H>1 / 2$. Caraballo et al. [6] investigated the existence, uniqueness, and exponential asymptotic behavior of mild solutions to stochastic delay evolution equations perturbed by a fractional Brownian motion with Hurst parameter $H>1 / 2$. Based on the research of [6], Boufoussi and Hajji [7] gave a proof of the existence and uniqueness of mild solutions for a neutral stochastic differential equation with finite delay, driven by a fractional Brownian motion in a Hilbert space. Dung [8] gave a sufficient condition for the exponential asymptotic behavior of solutions of a general class of linear fractional SDEs with time-varying delays. However, to the best of our knowledge, fractional SDEs with distributed delay have not been considered till now. In this paper, SDEs driven by an $\mathrm{fBm}$ with distributed delay will be introduced and asymptotic behavior of the solution will be analyzed.

In [2], Mandelbrot and Van Ness discussed the following integral representation:

$$
B^{H}(t)=B^{H}(0)+\frac{1}{\Gamma(H+1 / 2)}\left(Z_{t}+\int_{0}^{t}(t-s)^{H-1 / 2} \mathrm{~d} W(s)\right), \quad t>0,
$$

where

$$
Z_{t}=\int_{-\infty}^{0}\left[(t-s)^{H-1 / 2}-(-s)^{H-1 / 2}\right] \mathrm{d} W(s)
$$

$W(t)$ is a standard Brownian motion, $\Gamma$ represents the gamma function, and $0<H<1$ is the Hurst parameter. There are also several other stochastic integral representations for fBm [3].

As to the presentation introduced by Mandelbrot and Van Ness [2], the integral form

$$
W^{H}(t)=\int_{0}^{t}(t-s)^{H-1 / 2} \mathrm{~d} W(s)
$$

is called the Riemann-Liouville fractional integral. There is a relation between the Riemann-Liouville fractional Brownian motion (RLfBm) and fBm [9]. Considering that the process $Z_{t}$ has absolutely continuous trajectories, it suffices to consider the term $W^{H}(t)$ instead of $B^{H}(t)[10]$, thus, $B^{H}(t)$ will be denoted by $W^{H}(t)$ along our paper.

This paper is organized as follows: In Section 2, the explicit form of solution to the retarded SDE with an $\mathrm{fBm}$ is given. In Section 3, asymptotic behavior of the solution is provided, and sufficient conditions are derived to guarantee the $p$ th moment exponential stability and almost surely exponential stability.

\section{Preliminary and explicit solution}

In the following sections, we consider retarded SDE driven by an $\mathrm{fBm}$ in the form:

$$
\mathrm{d} X(t)=\left(\int_{-\tau}^{0} X(t+\theta) \rho(\mathrm{d} \theta)\right) \mathrm{d} t+\sigma(t) \mathrm{d} W^{H}(t), \quad t>0
$$

with the initial value $X(t)=\xi(t), t \in[-\tau, 0]$, where $W^{H}(t)$ is a RLfBm with Hurst parameter $H>\frac{1}{2}, \rho(\cdot)$ is a finite signed measure defined on $[-\tau, 0]$. 
In order to give the explicit form of (2.1), we first consider the following deterministic retarded differential equation:

$$
\mathrm{d} Y(t)=\left(\int_{-\tau}^{0} Y(t+\theta) \rho(\mathrm{d} \theta)\right) \mathrm{d} t
$$

with the initial value $Y(t)=\xi(t), t \in[-\tau, 0]$. It is easy to see that the characteristic equation of $(2.2)$ is

$$
h(\lambda):=\lambda-\int_{-\tau}^{0} e^{\lambda \theta} \rho(\mathrm{d} \theta)=0 .
$$

Denote $Z(t)$ as the fundamental solution of (2.2) with initial value $Z(0)=1$ and $Z(\theta)=0$, $\theta \in[-\tau, 0)$. By the variation-of-constants formula (see, e.g., [11]), the solution of (2.2) has a unique explicit form:

$$
Y(t)=Z(t) \xi(0)+\int_{-\tau}^{0} \int_{\theta}^{0} Z(t+\theta-s) \xi(s) \mathrm{d} s \rho(\mathrm{d} \theta), \quad t \geq 0 .
$$

According to Hale [12], for any $\alpha>\alpha_{0}:=\max \{\operatorname{Re}(\lambda): h(\lambda)=0\}$, there exists $k_{\alpha}>0$ such that the fundamental solution $Z(t)$ satisfies the inequality

$$
|Z(t)| \leq k_{\alpha} e^{\alpha t}, \quad t \geq-\tau .
$$

Before we introduce the explicit representation of (2.1), we first present a lemma that is useful in later parts.

Lemma 2.1 [13] For every $\epsilon>0$, denote by $\beta=H-\frac{1}{2}$, define

$$
W^{H, \epsilon}(t)=\int_{0}^{t}(t-s+\epsilon)^{\beta} \mathrm{d} W(s)
$$

Then $\left\{W^{H, \epsilon}(t)\right\}_{t \geq 0}$ is a semimartingale with the following decomposition:

$$
W^{H, \epsilon}(t)=\epsilon^{\beta} W(t)+\int_{0}^{t} \phi^{\epsilon}(s) \mathrm{d} s
$$

where $\phi^{\epsilon}(s)=\int_{0}^{s} \beta(s+\epsilon-u)^{\beta-1} \mathrm{~d} W(u)$. Furthermore, $W^{H, \epsilon}(t)$ converges to $W^{H}(t)$ in $L^{2}(\Omega)$ uniformly with respect to $t \in[0, T]$ when $\epsilon \rightarrow 0$.

Theorem 2.2 There is a unique strong solution $\{X(t)\}_{t \geq 0}$ to (2.1) which reads

$$
\begin{aligned}
X(t)= & Z(t) \xi(0)+\int_{-\tau}^{0} \int_{\theta}^{0} Z(t+\theta-s) \xi(s) \mathrm{d} s \rho(\mathrm{d} \theta) \\
& +\int_{0}^{t} Z(t-s) \sigma(s) \mathrm{d} W^{H}(s), \quad t \geq 0,
\end{aligned}
$$

where $Z(t)$ is the fundamental solution of (2.2) with initial value $Z(0)=1$ and $Z(\theta)=0$, $\theta \in[-\tau, 0)$. 
Proof According to Dung [8], the solution $X(t)$ of (2.1) can be approximated by solving the following equation:

$$
\mathrm{d} X^{\epsilon}(t)=\left(\int_{-\tau}^{0} X^{\epsilon}(t+\theta) \rho(\mathrm{d} \theta)\right) \mathrm{d} t+\sigma(t) \mathrm{d} W^{H, \epsilon}(t), \quad t>0
$$

with the initial datum $X^{\epsilon}(t)=\xi(t), t \in[-\tau, 0]$. By the decomposition (2.3), we can rewrite the above equation as

$$
\mathrm{d} X^{\epsilon}(t)=\left(\int_{-\tau}^{0} X^{\epsilon}(t+\theta) \rho(\mathrm{d} \theta)+\sigma(t) \phi^{\epsilon}(t)\right) \mathrm{d} t+\sigma(t) \epsilon^{\beta} \mathrm{d} W(t), \quad t>0 .
$$

Multiplying this equation by $e^{-\lambda t}, \operatorname{Re} \lambda>c$ sufficiently large, integrating from 0 to $\infty$, denoting by $\mathscr{L}\left(X^{\epsilon}\right)(\lambda)$ the Laplace transform of $X^{\epsilon}(t)$, we obtain

$$
\begin{aligned}
h(\lambda) \mathscr{L}\left(X^{\epsilon}\right)(\lambda)= & \xi(0)+\int_{-\tau}^{0} \int_{\theta}^{0} e^{\lambda(\theta-t)} \xi(t) \mathrm{d} t \rho(\mathrm{d} \theta)+\int_{0}^{\infty} e^{-\lambda t} \sigma(t) \phi^{\epsilon}(t) \mathrm{d} t \\
& +\int_{0}^{\infty} e^{-\lambda t} \sigma(t) \epsilon^{\beta} \mathrm{d} W(t) .
\end{aligned}
$$

An application of the Laplace inversion theorem (see, for example, [12]) yields

$$
\begin{aligned}
X^{\epsilon}(t)= & \int_{(c)} e^{\lambda t} h^{-1}(\lambda)\left[\xi(0)+\int_{-\tau}^{0} \int_{\theta}^{0} e^{\lambda(\theta-t)} \xi(t) \mathrm{d} t \rho(\mathrm{d} \theta)\right. \\
& \left.+\int_{0}^{\infty} e^{-\lambda t} \sigma(t) \phi^{\epsilon}(t) \mathrm{d} t+\int_{0}^{\infty} e^{-\lambda t} \sigma(t) \epsilon^{\beta} \mathrm{d} W(t)\right] \mathrm{d} \lambda .
\end{aligned}
$$

Note that

$$
\mathscr{L}\left(Z^{\epsilon}\right)(\lambda)=h^{-1}(\lambda)
$$

where $Z^{\epsilon}(t)$ is the fundamental solution of

$$
\mathrm{d} Y^{\epsilon}(t)=\left(\int_{-\tau}^{0} Y^{\epsilon}(t+\theta) \rho(\mathrm{d} \theta)\right) \mathrm{d} t
$$

with the initial value $Z^{\epsilon}(0)=1$ and $Z^{\epsilon}(\theta)=0, \theta \in[-\tau, 0)$.

With the definition of a convolution of the Laplace transform, we get

$$
\begin{aligned}
X^{\epsilon}(t)= & Z^{\epsilon}(t) \xi(0)+\int_{-\tau}^{0} \int_{\theta}^{0} Z^{\epsilon}(t+\theta-s) \xi(s) \mathrm{d} s \rho(\mathrm{d} \theta) \\
& +\int_{0}^{t} Z^{\epsilon}(t-s) \sigma(s) \phi^{\epsilon}(s) \mathrm{d} s+\int_{0}^{t} Z^{\epsilon}(t-s) \sigma(s) \epsilon^{\beta} \mathrm{d} W(s) .
\end{aligned}
$$

Therefore, by (2.3), it follows that

$$
\begin{aligned}
X^{\epsilon}(t)= & Z^{\epsilon}(t) \xi(0)+\int_{-\tau}^{0} \int_{\theta}^{0} Z^{\epsilon}(t+\theta-s) \xi(s) \mathrm{d} s \rho(\mathrm{d} \theta) \\
& +\int_{0}^{t} Z^{\epsilon}(t-s) \sigma(s) \mathrm{d} W^{H, \epsilon}(s), \quad t \geq 0 .
\end{aligned}
$$

Let $\epsilon \rightarrow 0$, the representation of $X(t)$ is obvious. 


\section{Exponential behavior}

The following two theorems provide the $p$ th moment exponential stability and almost sure exponential stability of the solutions to (2.1).

Theorem 3.1 Assume that $\alpha_{0}<0$, and there exists $\gamma>0$ such that

$$
\int_{0}^{\infty} e^{2 \gamma s}|\sigma(s)|^{2} \mathrm{~d} s<\infty
$$

then there exist positive constants $K$ and $\mu$ such that for each $p>0$, and the solution of (2.1) satisfies

$$
\mathbb{E}|X(t)|^{p} \leq K\|\xi\|_{\infty}^{p} e^{-\mu p t}, \quad t \geq 0,
$$

where $\|\zeta\|_{\infty}:=\sup _{-\tau \leq \theta \leq 0}|\zeta(\theta)|$.

Proof According to the explicit form (2.4), along with the inequality $|a+b+c|^{p} \leq k_{p}\left(|a|^{p}+\right.$ $\left.|b|^{p}+|c|^{p}\right), p, k_{p}>0$, it is easy to get

$$
\begin{aligned}
\mathbb{E}|X(t)|^{p}= & \mathbb{E} \mid Z(t) \xi(0)+\int_{-\tau}^{0} \int_{\theta}^{0} Z(t+\theta-s) \xi(s) \mathrm{d} s \rho(\mathrm{d} \theta) \\
& +\left.\int_{0}^{t} Z(t-s) \sigma(s) \mathrm{d} W^{H}(s)\right|^{p} \\
\leq & k_{p} \mathbb{E}\left(|Z(t) \xi(0)|^{p}+\left|\int_{-\tau}^{0} \int_{\theta}^{0} Z(t+\theta-s) \xi(s) \mathrm{d} s \rho(\mathrm{d} \theta)\right|^{p}\right. \\
& \left.+\left|\int_{0}^{t} Z(t-s) \sigma(s) \mathrm{d} W^{H}(s)\right|^{p}\right) \\
\leq & K_{1} e^{-\alpha p t}\|\xi\|_{\infty}^{p}+k_{p} \mathbb{E}\left|\int_{0}^{t} Z(t-s) \sigma(s) \mathrm{d} W^{H}(s)\right|^{p} .
\end{aligned}
$$

Denote by

$$
I(t):=k_{p} \mathbb{E}\left|\int_{0}^{t} Z(t-s) \sigma(s) \mathrm{d} W^{H}(s)\right|^{p} .
$$

We should remind the reader that $\mathrm{fBm}$ is a centered Gaussian process. So, before considering $I(t)$, we can give an estimation on $\left|\int_{0}^{t} Z(t-s) \sigma(s) \mathrm{d} W^{H}(s)\right|^{2}$ first. Applying (2.3), we obtain

$$
\begin{aligned}
& \int_{0}^{t} Z(t-s) \sigma(s) \mathrm{d} W^{H}(s) \\
& \quad=\lim _{\epsilon \rightarrow 0} \int_{0}^{t} Z(t-s) \sigma(s) \mathrm{d} W^{H, \epsilon}(s) \\
& \quad=\beta \int_{0}^{t} \int_{0}^{s} Z(t-s) \sigma(s)(s-u)^{\beta-1} \mathrm{~d} W(u) \mathrm{d} s \\
& \quad=\beta \int_{0}^{t} \int_{u}^{t} Z(t-s) \sigma(s)(s-u)^{\beta-1} \mathrm{~d} s \mathrm{~d} W(u) .
\end{aligned}
$$


By the Itô isometry formula and the Hölder inequality, we have

$$
\begin{aligned}
& \mathbb{E}\left|\int_{0}^{t} Z(t-s) \sigma(s) \mathrm{d} W^{H}(s)\right|^{2} \\
& \quad=\beta^{2} \int_{0}^{t}\left(\int_{u}^{t} Z(t-s) \sigma(s)(s-u)^{\beta-1} \mathrm{~d} s\right)^{2} \mathrm{~d} u \\
& \quad \leq \beta^{2} \int_{0}^{t}\left(\int_{u}^{t}|Z(t-s) \sigma(s)|^{2}(s-u)^{\beta-1} \mathrm{~d} s\right)\left(\int_{u}^{t}(s-u)^{\beta-1} \mathrm{~d} s\right) \mathrm{d} u \\
& \quad \leq t^{\beta} \int_{0}^{t}|Z(t-s) \sigma(s)|^{2} s^{\beta} \mathrm{d} s \leq t^{2 \beta} \int_{0}^{t}|Z(t-s) \sigma(s)|^{2} \mathrm{~d} s \\
& \quad \leq k_{\alpha}^{2} t^{2 \beta} \int_{0}^{t} e^{-2 \alpha(t-s)}|\sigma(s)|^{2} \mathrm{~d} s .
\end{aligned}
$$

By virtue of $\left[8\right.$, Lemma 3.1] and condition (3.1), letting $\gamma_{1}=\alpha \wedge \gamma$, we have

$$
\mathbb{E}\left|\int_{0}^{t} Z(t-s) \sigma(s) \mathrm{d} W^{H}(s)\right|^{2} \leq k_{\alpha}^{2} t^{2 \beta} e^{-2 \gamma_{1} t} \int_{0}^{\infty} e^{2 \gamma s}|\sigma(s)|^{2} \mathrm{~d} s \leq K_{2}^{2} e^{-2 \gamma_{2} t}
$$

where $K_{2}^{2}=k_{\alpha}^{2} t^{2 \beta} e^{-\gamma_{1} t} \int_{0}^{\infty} e^{2 \gamma s}|\sigma(s)|^{2} \mathrm{~d} s, \gamma_{2}=\frac{1}{2} \gamma_{1}$. Therefore, the Gaussian property leads to $I(t) \leq K_{2}^{p} e^{-\gamma_{2} p t}$. An application of $I(t)$ on (3.2) yields

$$
\mathbb{E}|X(t)|^{p} \leq K\|\xi\|_{\infty}^{p} e^{-\mu p t}, \quad t \geq 0
$$

where $\mu=\alpha \wedge \gamma_{2}$. This completes the proof.

Theorem 3.2 Assume that (3.1) is satisfied, then there exists a positive constant $v$ such that the solution of (2.1) has the property

$$
\limsup _{t \rightarrow \infty} \frac{1}{t} \log |X(t)| \leq-v
$$

Proof For $n-1 \leq t \leq n, n \geq 1, X(t)$ can be represented as

$$
X(t)=X(n-1)+\int_{n-1}^{t}\left(\int_{-\tau}^{0} X(s+\theta) \rho(\mathrm{d} \theta)\right) \mathrm{d} s+\int_{n-1}^{t} \sigma(s) \mathrm{d} W^{H}(s)
$$

With the inequality $|a+b+c|^{2} \leq 3\left(|a|^{2}+|b|^{2}+|c|^{2}\right)$, we derive from (3.2) that

$$
\begin{aligned}
\mathbb{E}\left(\sup _{n-1 \leq t \leq n}|X(t)|^{2}\right) \leq & 3 \mathbb{E}|X(n-1)|^{2}+3 \mathbb{E}\left(\int_{n-1}^{n}\left(\int_{-\tau}^{0} X(s+\theta) \rho(\mathrm{d} \theta)\right) \mathrm{d} s\right)^{2} \\
& +3 \mathbb{E}\left(\sup _{n-1 \leq t \leq n}\left|\int_{n-1}^{t} \sigma(s) \mathrm{d} W^{H}(s)\right|^{2}\right) \\
\leq & C_{1}\|\xi\|_{\infty}^{2} e^{-2 \mu(n-1)}+C_{2} e^{-2 \mu(n-1)}+3 J(t)
\end{aligned}
$$

where $J(t)=\mathbb{E}\left(\sup _{n-1 \leq t \leq n}\left|\int_{n-1}^{t} \sigma(s) \mathrm{d} W^{H}(s)\right|^{2}\right)$. 
Next, by the Burkhold-Davis-Gundy inequality, (2.3) and (3.1), we arrive at

$$
\begin{aligned}
J(t) & \leq \mathbb{E}\left(\sup _{n-1 \leq t \leq n}\left|\int_{n-1}^{t} \sigma(s) \mathrm{d} W^{H}(s)\right|^{2}\right) \\
& \leq C_{3} \int_{n-1}^{n}\left(\int_{u}^{n} \sigma(s) \beta(s-u)^{\beta-1} \mathrm{~d} s\right)^{2} \mathrm{~d} u \leq C_{4} e^{-2 \gamma(n-1)} .
\end{aligned}
$$

Let $\gamma_{3}=\mu \wedge \gamma$, we see that

$$
\mathbb{E}\left(\sup _{n-1 \leq t \leq n}|X(t)|^{2}\right) \leq C e^{-2 \gamma_{3} n}
$$

Then, with the Chebyshev inequality, for any $\gamma_{4}<\gamma_{3}$,

$$
\mathbb{P}\left(\sup _{n-1 \leq t \leq n}|X(t)|^{2}>e^{-2 \gamma_{4} n}\right) \leq e^{2 \gamma_{4} n} \mathbb{E}\left(\sup _{n-1 \leq t \leq n}|X(t)|^{2}\right) \leq C e^{-2\left(\gamma_{3}-\gamma_{4}\right) n} .
$$

Since $\sum_{n=1}^{\infty} e^{-2\left(\gamma_{3}-\gamma_{4}\right) n}<\infty$, by virtue of Borel-Cantelli lemma, there exists an $\Omega_{0} \in \Omega$ with $\mathbb{P}\left(\Omega_{0}\right)=1$ such that for every $\omega \in \Omega_{0}$, there exists an integer $n_{0}(\omega)$, for $n \geq n_{0}(\omega), n-1 \leq$ $t \leq n$,

$$
|X(t)|^{2} \leq e^{-2 \gamma_{4} n} \leq e^{-2 \gamma_{4} t}
$$

The desired conclusion is satisfied with $v=\gamma_{4}$.

Remark 3.1 This provides a method to ensure the exponential stability of SDEs driven by an $\mathrm{fBm}$, and it can be generalized to fractional SDEs of neutral type.

\section{Competing interests}

The author declares to have no competing interests.

\section{Author's contributions}

The author carried out the proof and conceived of the study. The author read and approved the final manuscript.

\section{Acknowledgements}

The author is very grateful to the editor and the anonymous referees for their insightful and constructive comments and suggestions, which have led to an improved version of this paper. The author also thanks the NNSF (71371193) for support.

Received: 20 September 2014 Accepted: 4 December 2014 Published: 18 Dec 2014

\section{References}

1. Kolmogorov, AN: Wienersche Spiralen und einige andere interessante Kurven im Hilbertschen Raum. C. R. (Dokl.) Acad. Sci. URSS 26, 115-118 (1940)

2. Mandelbrot, BB, Van Ness, JW: Fractional Brownian motions, fractional noises and applications. SIAM Rev. 10, $422-437$ (1968)

3. Biagini, F, Hu, YZ, Øksendal, B, Zhang, TS: Stochastic Calculus for Fractional Brownian Motion and Applications. Springer, London (2008)

4. Ferrante, M, Rovira, C: Stochastic delay differential equations driven by fractional Brownian motion with Hurst parameter $H>1 / 2$. Bernoulli 12, 85-100 (2006)

5. Ferrante, M, Rovira, C: Convergence of delay differential equations driven by fractional Brownian motion. J. Evol. Equ. $10,761-783(2010)$

6. Caraballo, T, Garrido-Atienza, MJ, Taniguchi, T: The existence and exponential behavior of solutions to stochastic delay evolution equations with a fractional Brownian motion. Nonlinear Anal. 74, 3671-3684 (2011)

7. Boufoussi, B, Hajii, S: Neutral stochastic functional differential equations driven by a fractional Brownian motion in a Hilbert space. Stat. Probab. Lett. 82, 1549-1558 (2012) 
8. Dung, N: Asymptotic behavior of linear fractional stochastic differential equations with time-varying delays. Commun. Nonlinear Sci. Numer. Simul. 19, 1-7 (2014)

9. Lim, SC, Sithi, VM: Asymptotic properties of the fractional Brownian motion of Riemann-Liouville type. Phys. Lett. A 206, 311-317 (1995)

10. Alòs, E, Mazet, O, Nualart, D: Stochastic calculus with respect to fractional Brownian motion with Hurst parameter lesser than 1/2. Stoch. Process. Appl. 6, 121-139 (2000)

11. Bao, JH, Yin, G, Yuan, CG: Stationary distributions for retarded stochastic differential equations without dissipativity. arXiv:1308.2018

12. Hale, JK: Theory of Functional Differential Equations. Springer, Berlin (1977)

13. Thao, TH: An approximate approach to fractional analysis for finance. Nonlinear Anal. 7, 124-132 (2006)

10.1186/1687-1847-2014-321

Cite this article as: Tan: Exponential stability of fractional stochastic differential equations with distributed delay. Advances in Difference Equations 2014, 2014:321

Submit your manuscript to a SpringerOpen ${ }^{\odot}$ journal and benefit from:

- Convenient online submission

- Rigorous peer review

- Immediate publication on acceptance

- Open access: articles freely available online

- High visibility within the field

- Retaining the copyright to your article 